\title{
Era of Quantum Computing- An Intelligent and Evaluation based on Quantum Computers
}

\author{
Shiny Irene D, G. Vamsi Krishna, Nalini M
}

\begin{abstract}
In today's world, the necessity for prime speed computing is extremely high that the classic computers area unit undoubtedly not sufficient. Because of the limitation of Newtonian mechanics, quantum technicalities are taking the position of game-changer in competition of calculation. Quantum computing is the study of quantum pc that works underneath the laws of quantum physics like tunneling, annealing, web, and superposition to complete tasks that take an enormous quantity of your time. During this paper we'll concisely see however quantum computers work and the way it will be employed in decrypting personal keys that the classic computers cannot reach during a short span of your time. One in all the most blessings of victimization quantum computers area unit that the work with efficiency and area unit $1000 \mathrm{X}$ times quicker than our classic computers.
\end{abstract}

Keywords: Quantum Computers, Quantum Computing, Quantum Inspired Evolutionary Algorithm, Quantum Bits, Quantum Entanglement and Quantum Superposition, Shor's Algorithm.

\section{INTRODUCTION}

For most of our history, humans are victimization their sticks, hearth and their brains to survive on the earth. As time went on sticks were restore by nuclear weapons and hearth by power plants however the largest upgrade since has been to our brain. Because of the upgrade, the technology that we tend to used became smaller and smaller. This procedure is getting ready to convene its material limits. A quantum pc may be a pc that works on the principles of quantum physics. The quantum realm is completely different thus this pc operates during a method that's ineffable within the real realm. In spite of the unbelievable energy procurable by supercomputers, there exist an entire cluster of issues that area unit still unscathed, as they can't answer them, by growing variety of data, we tend to area unit in large got to expand innovative tool to appear advance to look at or development the info, that area unit gift in massive amounts from the creation to human's polymer.

Revised Manuscript Received on October 21, 2019.

Shiny Irene D, Assistant Professor, Department of Computer Science and Engineering, Saveetha School of Engineering, Saveetha Institute of Medical and Technical Sciences, Chennai, India

G. Vamsi Krishna, Assistant Professor, Department of Computer Science and Engineering Dr.Lankapalli Bullayya Engineering College for Women, New Resupuvanipalem, Visakhapatnam

Nalini M, Assistant Professor, Department of Computer Science and Engineering, Saveetha School of Engineering, Saveetha Institute of Medical and Technical Sciences, Chennai, India

\section{QUANTUM COMPUTING}

Quantum computation is that the observation and analysis of computers and machines that use quantum laws and quantum physics for computation of information and processes. It's specialized in the quantum values of the given knowledge and converts them into specialized bits known as the quantum bits in contrast to binary bits in classical computers. The quantum computation has recently picked up pace because the applications and its ability has become additional clear to the globe. This might eventually result in additional development during this field and increase the pertinence in our way of life. The quantum pc uses the quantum rotation/orientation and superposition of electrons to store and method knowledge. This makes it additional viable as additional knowledge will behold on within the variety of negatron and it works as quick because of the speed of sunshine.

\section{A. Why classical computers will reach their end}

A pc machine may be a typical organization of hardware elements that area unit wont to perform tasks in line with the instruction given by the user. It performs tasks like info and displaying knowledge in line with user's need, process it victimization given instruction theme, and making management methods for internal hardware in line with the given directions. A pc is formed from straightforward modules that perform bound actions. These modules area unit created from a pc chip that consists of a variety of transistors. A junction transistor is what that processes the info during a pc, a switch which will conjointly finish or begin the flow of information. This knowledge is formed from bits which might be found to either one or zero. Advanced info will be portrayed by many permutations of those bits. Even the mixture of those transistors performs basic operations. By combining some of those gates we can produce some purposeful modules to perform operations, for instance, to add. Once you'll add, you'll multiply, and once you'll multiply, you'll essentially do something. However, once elements become smaller we tend to slowly shake the $\$ 64000$ realm and enter the quantum realm wherever things area unit a small amount completely different. A junction transistor will be assumed to associate degree electric switch and electricity is electrons moving between completely different points. So, a switch may be an electronic equipment connection-component that enables the movement of electrons during a circuit counting on the state of the switch. Today, the scale of a transistors is fourteen $\mathrm{nm}$, that is regarding eight times but the HIV' diameter, and 
five hundred times smaller than a red somatic cell. As transistors area unit continued to shrink to a size not larger than some atoms, electrons may transfer themselves to the opposite facet of a blocked passage via a method known as Quantum Tunneling. Within the quantum realm, ancient computers can simply stop creating sense. This can be a true physical barrier for our technological progress. To resolve this drawback, we tend to area unit victimization quantum properties to our advantage to make quantum computers.

\section{B. How do quantum computers work}

In traditional computers we tend to use bit, that area unit the tiniest unit of data. Similarly, the quantum computers use -qubit that conjointly represent one in all 2 values. A qubit will be any 2 level quantum system like a spin and a magnetic flux or an individual gauge boson. The 2 potential states of the system area unit zero and one just like the photons horizontal or vertical polarization. Within the quantum world, the qubit doesn't have to be only one of these; it will be in any proportions of each the states quickly. This can be known as superposition.

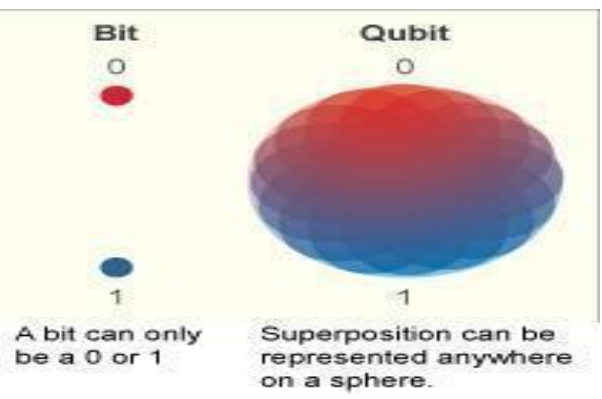

\section{Fig. 1 Structure of Bit and Qubit}

By Copenhagen's interpretation of the Schrödinger's cat equation, once determined, this qubit ought to collapse into either zero or one i.e. it will ne'er be each once determined. Therefore as long as it's unobserved, the qubit is in superposition of chances for zero and one, and you can't predict that it will be Four classical bits will be in on of $2 n$ completely different configurations at a time. That's sixteen potential mixtures, out of that you'll use only one. Four qubits in superposition, however, will be altogether those sixteen mixtures quickly. This variety grows exponentially with every additional qubit. Twenty of then will already store $1,000,000$ values in parallel.

One property exhibited by qubits is web, a detailed association that produces every of the qubits reach to every modification within the other's state outright, despite however so much apart. This implies that once mensuration only one entangles qubit, you'll directly deduce properties of its partners.

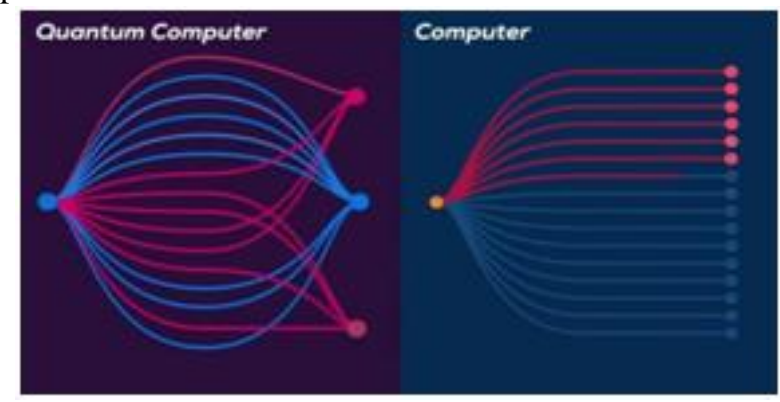

Fig. 2 Difference between Quantum Computer and Computer

So quantum computers sets up some qubits, applies quantum gates to entangle them and manipulate chances, the final live the outcomes, collapsing superposition to associate degree actual sequence of $0 \mathrm{~s}$ and $1 \mathrm{~s}$. This implies that you simply get the whole calculations that area unit potential with you set up, all done at constant time.

Ultimately, you'll solely live one in all the results associate degree it'll solely most likely be the one you wish, therefore you will get to check and check out once more. By victimization superposition and web, this could be exponentially additional economical than would ever be potential on a standard pc.

\section{Use of quantum computers to ruin IT security}

Today, your browsing, email, associate degreed banking knowledge is being unbroken secure by an encoding system during which you provide everybody a public key to encrypt messages solely you'll decipher. The matter is that this public key will truly be wont to calculate your secret personal key. Don't panic as computing this personal key will take years along on a standard pc. However, a quantum pc with exponential speed-up will do constant in seconds!

\section{The math behind it}

One of the most strategies of cryptography, the secret writing and cryptography secure communications, uses very massive prime numbers. As we know, it's pretty straightforward to search out massive prime numbers and multiply them along, however, it's very arduous for a pc to try and do the opposite- notice the prime factors of a very massive variety. The prime factors of variety area unit all the prime numbers that equally divide it. Normally, RSA cryptography uses these prime factors like keys to decode messages. therefore if you wish to listen in, you'll get to notice one in all these keys to hack in this is, you'll get to notice the print factors of a giant variety ( as in many digits long ). For instance let's take a little variety, 35 . The prime factors of thirty-five area unit five and seven. This can be found by checking all the prime numbers below thirty-five.

Table. 1 Impact Examination of Quantum Computing on Encryption Methods

\begin{tabular}{|l|l|l|l|}
\hline $\begin{array}{l}\text { Cryptographic } \\
\text { Algorithm }\end{array}$ & Type & Purpose & $\begin{array}{l}\text { Impact From } \\
\text { Quantum Computer }\end{array}$ \\
\hline AES-256 & Symmetric key & Encryption & Secure \\
\hline SHA-256, SHA-3 & - & Hash functions & Secure \\
\hline RSA & Public key & $\begin{array}{l}\text { Signatures, key } \\
\text { establishment }\end{array}$ & No longer secure \\
\hline $\begin{array}{l}\text { ECDSA, ECDH(Elliptic } \\
\text { Curve Cryptography) }\end{array}$ & Public key & Signatures, key exchange & No longer secure \\
\hline $\begin{array}{l}\text { DSA(Finite Field } \\
\text { Cryptography) }\end{array}$ & Public key & Signatures, key exchange & No longer secure \\
\hline
\end{tabular}


This is a very time overwhelming method once it involves large numbers. Therefore we'd like a higher strategy to search out the prime factors of very massive prime numbers. Here is wherever one in all the nice scientific discipline heroes, Euler, involves the rescue. He found standard arithmetic that is essentially all the mathematics underlying RSA cryptography. Standard arithmetic is essentially counted during a circle. Example count Mod three would be zero, $1,2,0,1,2,0,1,2 \ldots$ In straightforward it's the rest once dividing 2 numbers. We tend to use this rotary relying on arithmetic operations. After we take $\bmod 10$ on the $3 \mathrm{k}$ sequence.

From the higher than observation because the sequence of powers simply gets larger and greater, however, the standard version of the sequence cycle i.e. they repeat constant patter over and once more. Another factor which will be determined in this for any such series the last term of the cycle is usually one.

\section{Table. 2 Example Cyclical Counting}

\begin{tabular}{|c|c|}
\hline 3k & 3mod10 \\
\hline 3 & 3 \\
\hline 9 & 9 \\
\hline 27 & 7 \\
\hline 81 & 3 \\
\hline 243 & 9 \\
\hline 729 & 7 \\
\hline 2187 & 1 \\
\hline 6561 & \\
\hline
\end{tabular}

Hence as long as $\mathrm{x}$ and $\mathrm{n}$ area unit relatively prime $\mathrm{x}$ mod $n, x 2 \bmod n, x 3 \bmod n, x 4 \bmod n \ldots$ can have this property. we tend to decision the length of the continuance patter an amount. Therefore the amount \{of three/of three $\}$ mod ten is four and therefore the amount of two mod seven is 3 etc.

So why is that the amount important? If the amount of $\mathrm{x}$ mod $\mathrm{n}$ is a few variety $\mathrm{r}$, then $\mathrm{r}$ is that the smallest variety such $\mathrm{x} \equiv$ one $\bmod \mathrm{N}$., for instance, thirty-four $\equiv$ one mod ten. Therefore what will that got to do with factorization massive numbers? Let $\mathrm{N}$ be variety such $\mathrm{N}=\mathrm{p} *$ letter of the alphabet wherever $\mathrm{p}$ and letter of the alphabet area unit 2 prime numbers. to search out $\mathrm{p}$ and letter of the alphabet is that the goal.

Step 1:-

Pick any number smaller than N.a

(Check to see that a and $\mathrm{N}$ are relatively prime by computing the GCD. If the GCD is 1 then they are naturally relatively prime)

\section{Step 2:-}

Compute the period of a mod N.r

$\left(* \mathrm{r}\right.$ should be even $\left.* \mathrm{a}^{2 / 1}+1 ! \equiv 0 \bmod \mathrm{N}\right)$

\section{Step 3:-}

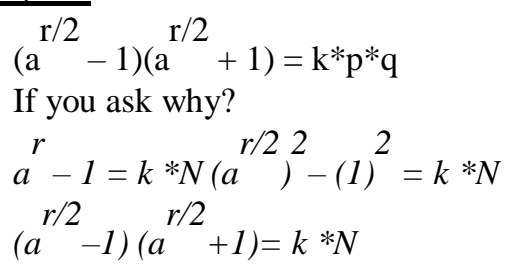

$$
\left(a^{r / 2}-1\right)\left(a^{r / 2}+1\right)=k * p * q
$$

STEP 4:-

Solve for $\mathrm{p}$ and $\mathrm{q}$

$$
p=\operatorname{GCD}\left(a^{r / 2}-1, N\right) q=\operatorname{GCD}\left(a^{r / 2}+1, N\right)
$$

The above algorithm is the outline of the Shor's algorithm and can be represented in a flowchart format by,

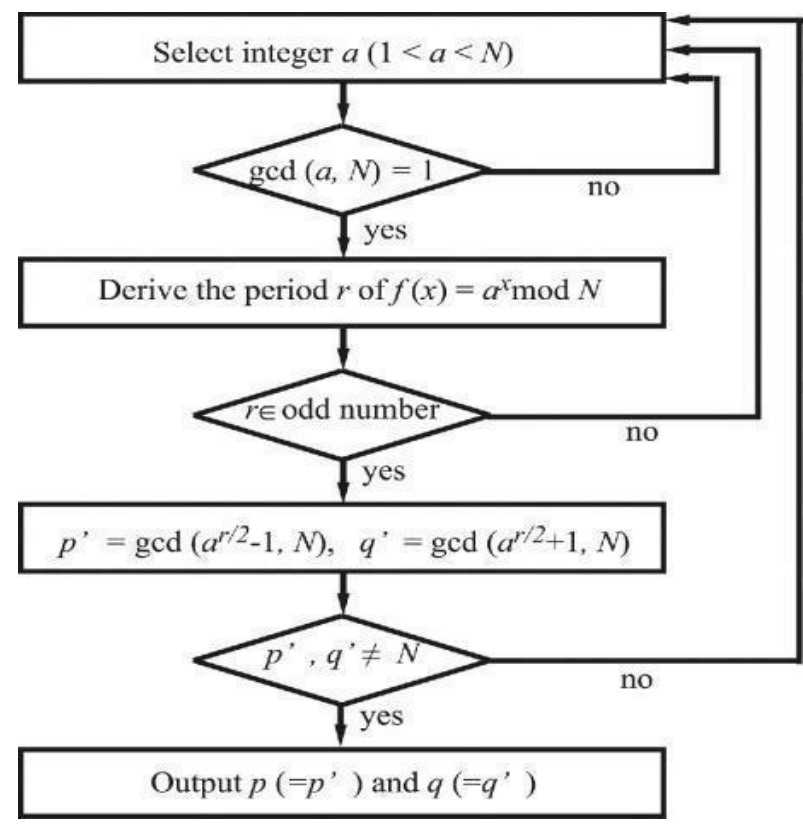

Fig. 3 Flow Chart of Shor's Algorithm

Now here's the catch, step 2, finding the amount takes an extended time. Therefore now's wherever quantum computing comes in. A quantum pc produces of these potential answers at constant time. Though' it looks like a sort of a bunch of classical computers operating in parallel, that's not the case. If it were, a quantum pc is during a superposition of basis states, that area unit the type of states a classical pc may be in. Since a superposition may be a combination of basic states and there's some chance related to perceptive them. to search out that chance, you sq. the amplitude of the amount ahead of the essential state. Therefore quantum pc isn't truly altogether these states. It simply splits itself into these many various items. After you arouse the results of a computation, it doesn't tell you regarding all $\mathrm{N}$ items it's in. it'll choose a state and tell you what the state says. If it were several pc connected parallel to every aside from the chance of the chosen state is not a reality is higher. To harness the facility of quantum computers, we'd like every of those basic states and therefore the elements of the superposition to be operating along. By a theoretical scientist Scott Aaronson, quantum reasons cannot compute advanced search sequences simply by applying brute force to render answers.

So however can we do it? we'd like to utilize the properties of its entire superposition and not simply a few|a couple of|a variety of|some|many\} of its basic states to try and do that Shor's algorithmic rule uses higher than found number theory, 
to remodel the matter of finding the factors of a given variety into finding a special variety, the amount of a selected periodic operate. We start with $\mathrm{n}$ completely different states representing the numbers one through n. For every state we tend to reason ax mod $n$, wherever $\mathrm{x}$ is that the variety of the state. Therefore, currently the states area unit a $\bmod n, a 2 \bmod n$, a3 $\bmod n$, and so on.

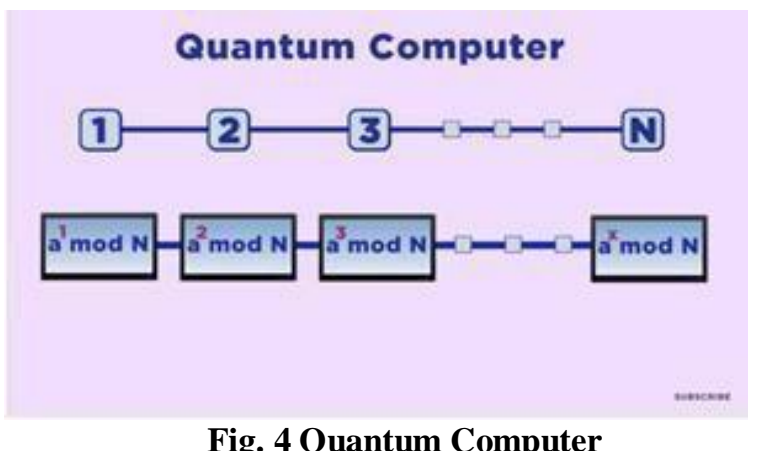

Then we tend to simply explore for the tiniest thereon says one then we're done. However, we tend to can't simply scan all the states. After we explore the results of a quantum computation, it simply shows one random state. The amount may be a world property of this quantum superposition. It's not a reality regarding one or 2 of those basic states. It's a reality regarding the whole wave of numbers created by superposition, however, usually, it repeats. That's the amount. We tend to apply one thing referred to as the quantum Fourier remodel to the mod series. The QDT uses resonances to amplify the essential state related to the right amount, and therefore the incorrect answer destructively interfere that suppress their amplitude. This could be compared with the noise cancelation technique use in headphones. This amplifies the chance of the right amount. This can be achieved by adding the advanced roots of unity with area unit essentially what the QDT will. Thus the 2 prime numbers area unit found and therefore the system has been compromised.

\section{RESULT}

Hence quantum computers area unit won't to decipher the general public and personal keys of a given network request price tag and thus forth used for constructive observation and analysis of information that floats around the web. Due to its high speed and potential, this technology can even be employed by government agencies to watch software engineer activities and take needed actions and measures. The whole potential and talents of quantum computers haven't been nevertheless discovered. Additional usage of those machines would facilitate us to enhance on this preexisting technology and work additional with efficiency, sound on the unturned potentialities of people in general.

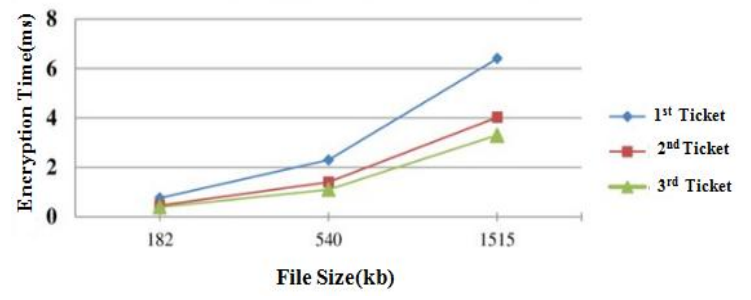

Fig. 5 Key Encryption Time

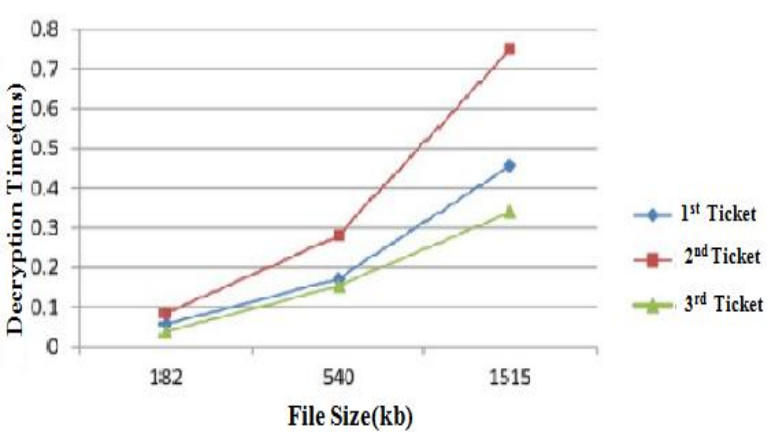

Fig. 6 Key Decryption Time

\section{CONCLUSION}

Quantum computers area unit successive large factor as what the human mind craves for the foremost, advancement, cannot be achieved while not it. At the appearance of quantum all the principles, algorithms, encryption, machine techniques can modification facultative US to use straightforward algorithms to realize nice heights. It'll not solely compromise as of information that flows through the web however will produce a brand new era of technology, associate degree era of the quantum realm.

\section{REFERENCES}

1. M. Dusek, N. L “ utkenhaus, and M. Hendrych, "Quantum cryptography," . Progress in Optics, vol. 49, pp. 381-454, 2006.

2. C. Paar and J. Pelzl, "Introduction to Public-Key Cryptography," in Understanding Cryptography. Berlin, Heidelberg: Springer Berlin Heidelberg, 2010, pp. 149-171.

3. Z. Kirsch, "Quantum Computing: The Risk to Existing Encryption Methods," Ph.D. dissertation, Tufts University, Massachusetts, 2015,

4. M. A. Nielsen and I. L. Chuang, Quantum Computation and Quantum Information: 10th Anniversary Edition, 10th ed. New York, NY, USA: Cambridge University Press, 2011

5. Nalini, M. and Anbu, S., "Anomaly Detection Via Eliminating Data Redundancy and Rectifying Data Error in Uncertain Data Streams", Published in International Journal of Applied Engineering Research (IJAER), Vol. 9, no. 24, 2014.

6. R. Jozsa, "Entanglement and Quantum Computation," in Geometric Issues in the Foundations of Science, S. Huggett, L. Mason, K. Tod, S. Tsou, and N. Woodhouse, Eds. Oxford University Press, July 1997.

7. W. Tichy, "Is quantum computing for real?: An interview with catherine mcgeoch of d-wave systems," Ubiquity, vol. 2017, no. July, pp. 2:1-2:20, Jul. 2017. [Online]. Available: http: //doi.acm.org/10.1145/3084688

8. Nalini, M. and Anbu, S., "A Novel Framework for Automatic Data Maintenance for DBMS Development", Published in Australian Journal of Basic and Applied Sciences (AJBAS), Vol. 9, no. 36, pp.198-206, 2015

9. M. Soeken, T. Haner, and M. Roetteler, "Programming quantum com- " puters using design automation," arXiv preprint arXiv:1803.01022, 2018.

10. S. Bone and M. Castro, "A Brief History of Quantum Computing," Surveys and Presentations in Information Systems Engineering (SURPRISE), vol. 4, no. 3, pp. 20-45, 1997, http://www.doc.ic.ac.uk/ nd/ surprise 97/journal/vol4/spb3/.

11. J. Muhonen and T. Dehollain, "Storing Quantum Information For 30 Seconds In a Nanoelectronic Device," Nature Nanotechnology, vol. 9 , pp. 986-991, 2014.

12. Nalini, M. and Uma Priyadarsini, To Improve the Performance of Wireless Networks for Resizing the Buffer, Proceedings of the 2019 international IEEE Conference on Innovations in Information and Communication $\quad$ Technology, $\quad$ Apr 2019 [DOI>10.1109/ICIICT1.2019.8741406] 
13. D-Wave, "Quantum Computing: How D-Wave Systems Work," http: //www.dwavesys.com/our-company/meet-d-wave.

14. L. S. Bishop, S. Bravyi, A. Cross, J. M. Gambetta, and J. Smolin, "Quantum volume," Technical report, 2017., Tech. Rep., 2017.

15. M. Campagna and C. Xing, "Quantum Safe Cryptography and Security: An Introduction, Benefits, Enablers and Challenges," ETSI, Tech. Rep. 8, 2015.

16. W. Buchanan and A. Woodward, "Will Quantum Computers be the End of Public Key Encryption?" Journal of Cyber Security Technology, vol. 1, no. 1, pp. 1-22, 2016.

17. Uma Priyadarsini and Nalini, M, Transient Factor- Mindful Video Affective Analysis- A Proposal for Internet Based Application, Proceedings of the 2019 international IEEE Conference on Innovations in Information and Communication Technology, Apr 2019. [DOI>10.1109/ICIICT1.2019.8741466]

18. L. Chen, S. Jordan, Y.-K. Liu, D. Moody, R. Peralta, R. Perlner, and D. Smith-Tone, "NIST: Report on Post-Quantum Cryptography," NIST, Tech. Rep., 2016

19. P. W. Shor, "Algorithms for Quantum Computation: Discrete Logarithms and Factoring," in Proceedings of the 35th Annual Symposium on Foundations of Computer Science, ser. SFCS '94. Washington, DC, USA: IEEE Computer Society, 1994, pp. 124-134.

20. Padmanaban and Nalini, M. , Adaptive Fuel Optimal and Strategy for vehicle Design and Monitoring Pilot Performance, Proceedings of the 2019 international IEEE Conference on Innovations in Information and $\begin{array}{llll}\text { Communication } & \text { Technology, } & \text { Apr } & 2019 \text {. }\end{array}$ [DOI>10.1109/ICIICT1.2019.8741361] 